\title{
Extraction of Region of Interest (ROI) for Palm Print and Inner Knuckle Print
}

\author{
M.L. Anitha \\ Research Scholar, \\ P.E.T. Research Center \\ P.E.S. College of Engineering, \\ Mandya, Karnataka, India.
}

\author{
K.A. Radhakrishna Rao, PhD \\ Professor, \\ Department of ECE, \\ P.E.S. College of Engineering, \\ Mandya, Karnataka, India.
}

\begin{abstract}
This paper proposes an novel approach to extract the Region of interest (ROI) for palm print and inner knuckle print(IKP) from hand images captured from a digital camera. An new algorithm to detect candidate key points on hand region is described. Using the candidate key points a novel approach to locate the ROI of palm print and inner knuckle print is proposed. The proposed approach is evaluated using the database collected at our institute. The results obtained are promising and confirm the usefulness of proposed ROI extraction approach for developing hand based biometric recognition systems.
\end{abstract}

\section{Keywords}

Biometric systems, ROI, Palm print, IKP.

\section{INTRODUCTION}

In recent years, biometric based recognition systems have been widely used as an effective method for automatically recognizing a person's identity since traditional recognition systems which are based on what a person knows(passwords) or what a person has(tokens) are not reliable due to use of several advanced techniques of forgery and password hacking. Biometric recognition is based on physiological traits such as finger print, palm print, iris, face and behavioral traits such as gait or signature associated with the person. Since each of these biometric traits have their own trait specific issues like pose, illumination, occlusion, rotation, translation and user acceptance one cannot consider any trait as best so that it suits for all types of applications. Use of Hand related biometric traits for developing recognition systems have gathered more attraction because of their inexpensive acquisition devices and high user acceptance. Biometric system consists of two subsystems; one for enrollment and second one for recognition. In the enrollment stage, biometric data are acquired from the individuals, feature sets are extracted from the acquired data, and one or more templates per individual are computed and stored in database. In the recognition stage biometric systems can operate in two modes, Verification or Identification[1-3].Verification refers to confirming or denying a person's claimed identity. In this mode the system performs one to one comparisons of the template computed from the acquired biometric data with the individual's own biometric templates stored in the database. Identification refers to establishing a person's identity. In this mode, template computed from the acquired user's biometric input is compared with the templates of all persons enrolled in the database to establish an individual identity.

Figure 1 shows a biometric recognition system. It contains four main modules which are data acquisition module, preprocessing module, feature extraction module and matching module.

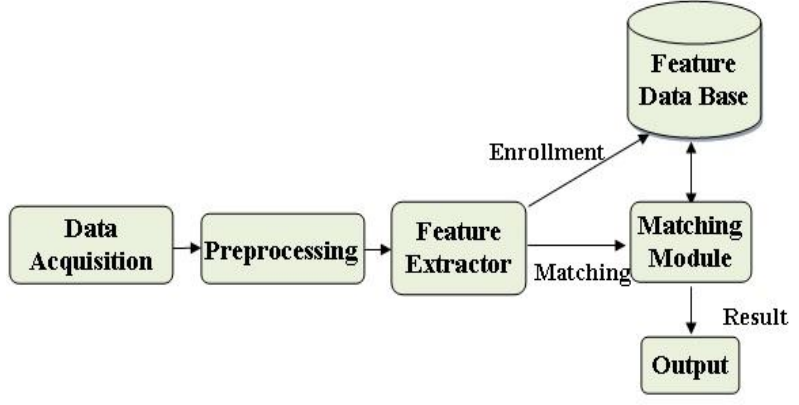

Figure 1 Biometric recognition system

1. Data acquisition module -This module is responsible for acquiring raw biometric data in the form of images. Majority of the acquisition modules utilizes a scanner or camera.

2. Preprocessing module- This module transforms acquired raw biometric data into a form that is suitable for feature extraction. ROI is cropped from input data. Depending on the trait, different algorithms are applied to extract the ROI.

3. Feature extraction module-From the preprocessed image, a set of salient or discriminatory features are extracted which represents the biometric trait and are stored as templates in database.

4. Matching module-This module matches the extracted feature vector of test image (probe template) against templates of enrollees present in the database (gallery set templates) to generate matching score. This module also encapsulates a decision making module which confirms the claimed identity (verification) or establishes the identity (identification).

Among all the hand related modalities deployed in recent years IKP and palm print modalities are selected because of its advantages. First, data acquisition is relatively easy since acquisition can be done in less constrained environment. Second both the modalities can be extracted from palmer surface of hand. Third both the modalities can be acquired without the need to touch any platform or hold any guidance for hand placement. Finally only one acquisition set up to capture palmer surface of hand is required. Palm print refers to the visible principal lines, wrinkles and ridges on palmer surface of hand region. IKP is the image patterns of skin folds and creases on the inner finger knuckle surface also present on palmer region of hand

The contribution of this paper are as follows. An image acquisition scheme is designed to capture the hand image and novel algorithm is developed to detect the ROI of palm print and IKP. 
The rest of the paper is organized as follows. Section 2 presents a brief review of research on ROI extraction. In section 3 proposed approach for ROI extraction along with steps are explained. The effectiveness of the ROI approach has been tested with a texture based verification system and results obtained are discussed in section 4 . Finally conclusions are given in section 5 .

\section{RELATED WORK}

Acquisition of hand related biometric traits have evolved from early approaches which considers flat surface and pegs to guide the placement of user's hand to completely platform free and contact free acquisition set up. Based on the image acquisition criteria acquisition set up are classified into following three categories

1. Constrained and contact based- Flat platform and pegs to restrict hand position during image acquisition.

2. Unconstrained and contact based- Flat plat form with no guidance pegs to restrict the user's hand position during image acquisition.

3. Unconstrained and contact free- Platform free and contact less set up where neither pegs nor platform are used for hand image acquisition.

For the first two categories individuals must place their hand on the sensor or a flat surface with or without pegs which makes the individuals to feel very uncomfortable during image acquisition and causes sanitary issues in public areas. These reasons greatly limit the applicability of the recognition systems developed using these type of acquisition set up for various commercial and civilian applications. Hence there exist greater demand for design of new types of biometric systems which uses unconstrained and contact free image acquisition set up. In this section different methods proposed by researchers for finding palm print and IKP ROI are discussed for image capturing systems that works without using pegs.

In [4] authors have captured hand images from 170 individuals using a CCD camera without any pegs for hand placement. Valley points between fingers are used as reference points to align the hand image such that palm region has minimal rotation and translation errors. The maximum square region that can fit the palm area is selected as ROI of palm print and the square region is centered on the axis running through the gap between middle finger and ring finger.

In [5] authors have captured hand images from 50 individuals using a flat bed scanner. The central line of middle finger is considered as principal axis in locating the palm print ROI. A fixed size ROI is extracted from the palm region.

A scanner platform is used by Tee connie et al. in [6] to capture hand images. Two mid points, one at the bottom finger region of index finger and another at bottom finger region of little finger are computed to form the base line. A square region with base line found as one side is cropped as ROI of palm print.

ROI extraction in [7] by Zohaib Khan et al. is based on the two valley points identified one between middle and index finger and another between ring and little finger. The line joining the two valley points is considered as one side of ROI region and another line parallel to it at $2 / 3^{\text {rd }}$ of distance from valley points is considered as another side of ROI region. End points of these two lines are considered as four corners of ROI region.

Radial distance from centre of wrist is used to locate the valley points between fingers and tip points of individual fingers by Zhu le Qing et al. in [8]. The line joining the tip of little finger to midpoint of little finger base region is extended by twenty percent the length of little finger to fix one point of ROI region Similarly one more ROI point is computed using index finger and the line joining these two ROI points are considered as one edge. This edge is used to crop a square region which is considered as palm print ROI.

Web camera is used by Goh Kah et al. in [9] to capture hand images. Authors have proposed competitive hand valley detection algorithm for locating four valley points between all five fingers. Further projectile search method is proposed by the authors to find the finger tips using the valley points. Identified valley points are used in extraction of palm print ROI and finger tips points are used to extract the largest rectangle area lying inside the contour of the finger which is considered as IKP ROI.

In [10] Qiang $\mathrm{Li} \mathrm{Q}$ et al. have proposed an personal identification system based on IKP by capturing hand images using a digital camera. Authors have cropped the middle finger form the hand image by finding datum points. By applying randon projection on middle finger the knuckle print around second knuckle of middle finger is cropped as IKP ROI.

IKP ROI's of all fingers except thumb is extracted from hand image captured by cameras equipped on portable devices by Xuemiao $\mathrm{Xu}$ et al. in [11]. Finger axis is defined as the line passing through the centre region of the finger. The finger axis is aligned to horizontal direction and the middle knuckle located between $1 / 2$ to $3 / 4$ the length of the finger is extracted as IKP ROI using randon transformation.

\section{PROPOSED APPROACH}

When hand images are captured without using any pegs the position, direction and amount of stretching of a palm region of hand may vary. Also hand image acquired from the same palm may have little rotation due to orientation in hand placement and translation during image acquisition. Furthermore, palm size varies from one individual to another individual. Hence acquired hand images without any constraints needs to be preprocessed such that the extracted $\mathrm{ROI}$ is robust to translation and orientation of hand placement. The overall ROI extraction approach is shown in figure 2 .

\subsection{Data acquisition}

A data acquisition set up is designed to acquire hand images. A very user friendly acquisition has been set up for the collection of images. The system uses a camera mounted on a tripod stand and focused against a black panel under normal room lightning conditions. The camera is placed at a suitable distance from the black panel and the user's hand can be placed in front of the panel so that their hand image can be captured. The capture procedure is peg free which is well acceptable by users. The only requirement for hand placement 


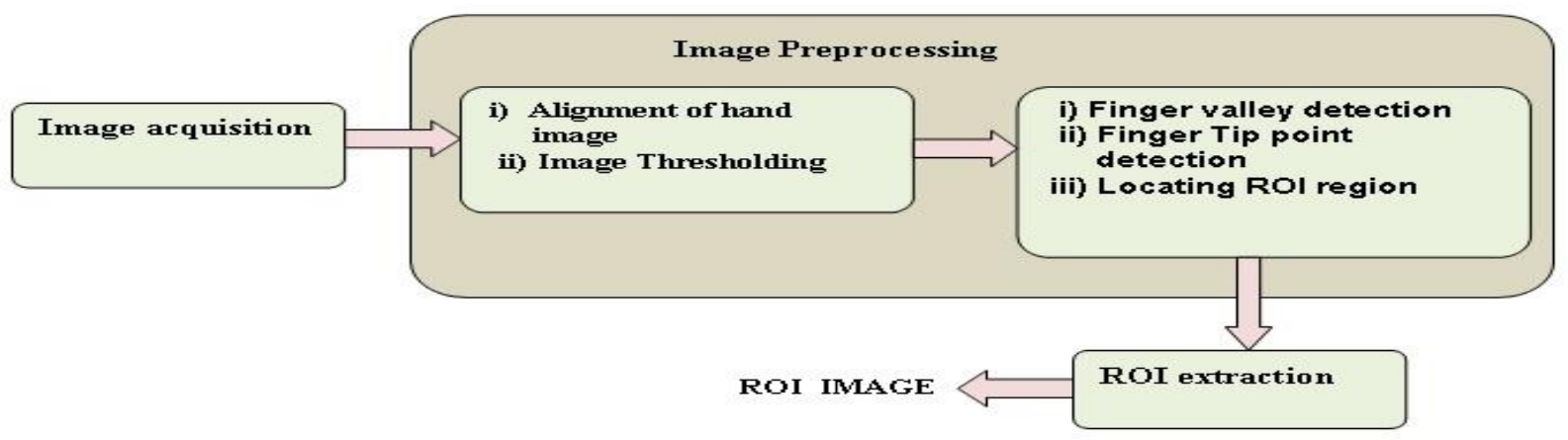

Figure 2 ROI Extraction process

is that the fingers must be placed such that there is space between two adjacent fingers. Also users are requested to keep their hand orientation straight. If there is change in the hand posture either in clockwise or anticlockwise direction by around 45 degrees, alignment is done in the preprocessing stage.

Our data collection process was carried out at two different locations which had no restrictions on surrounding illuminations. The data collection process spanned over three months and 448 persons volunteered for the database. The participants were mainly students from our institute and were in the age group of 20 to 24 years. Four right hand images of each user were captured in two sessions resulting in eight images of each user in the database with a total of 3,584 images in the database. Sample of the captured image is shown if figure 3 .

\subsection{Image preprocessing}

Preprocessing steps consist of three stages. First we segment the hand image from background. After that finger tips and hand valley detection algorithm is used to find the tip points of little, ring, middle and index fingers and valley points between adjacent fingers. These tip points and valley points serve as base points to find finger IKP ROI location and palm print ROI location. The processing steps are provided in the following sections.

\subsubsection{Alignment of hand image and thresholding} Initially we find two points on left edge of palm region by using the following steps

i) A line path is traced from left edge of captured image following a horizontal path till palm region is found.

ii) One more line path is traced following a horizontal path fifty pixels above the first line path till a hand region is found. This process is illustrated in figure 4.

iii) Slope of the line joining these two points is computed and the complete hand region is rotated either in clockwise or anticlockwise direction so that the orientation of left edge of palm region becomes straight. This is illustrated in figure 5. This rotated image is considered for further preprocessing process.

The steps for thresholding the hand image to get binary image are as follows

i) Captured color image is converted to gray level image since gray level image is adequate for extracting the features.

ii) A low pass Gaussian filter is applied to remove the noise pixels from gray level image. iii) A fixed threshold is applied to convert the gray level image into a binary image.

\subsubsection{Finger valley and tip detection}

An novel finger tip and valley detection algorithm is proposed. The proposed algorithm traverses the contour of palm image to find possible tip and valley locations. Rather than scanning the complete hand contour, all points below the centre of hand region are discarded since tip and valley points lies above the centre of hand region. This process greatly speeds up the detection process. A point on contour is qualified as a point of interest if it has some neighboring points lying in the non hand region while majority of neighboring points are in the hand region. Based on this fact conditions are formulated to find the valley point. Points must satisfy all the conditions to consider it as valley point, if it fails to satisfy any one condition, the current point will be rejected and the algorithm proceeds to check next point along the hand contour. Three valley points are identified. First point between little finger and ring finger, second one between ring and middle finger and the third point between middle finger and index finger.

The conditions to check current point for valley existence are

Condition 1. Eight points which are five pixels away from current point $(p)$ are considered. These points are selected such that they are at equal distance from current pixel. If there are at least one and not more than three consecutive points falling in the non hand region while remaining points within hand region, then $\mathrm{p}$ is considered as candidate for valley and algorithm proceeds to check second condition. Otherwise the algorithm continues to check the next point along hand contour.

Condition 2. Considering $\mathrm{p}$ as centre of circle and using midpoint circle generation algorithm points along the circumference of circle with radius ten are found. If number of points lying in the hand region is more than thirty percent of the total points on the circumference of the circle then it is considered as valley point. Otherwise the algorithm continues to check the next point along hand contour.

\subsubsection{Valley detection Algorithm}

Algorithm 1: Input: Binary image. Output: Valley points

Step 1 Contour of the hand image is obtained from the binary image and centre of hand region is computed. This is illustrated in figure 6 .

Step 2 Points along hand contour which are above the center of hand region are considered. 


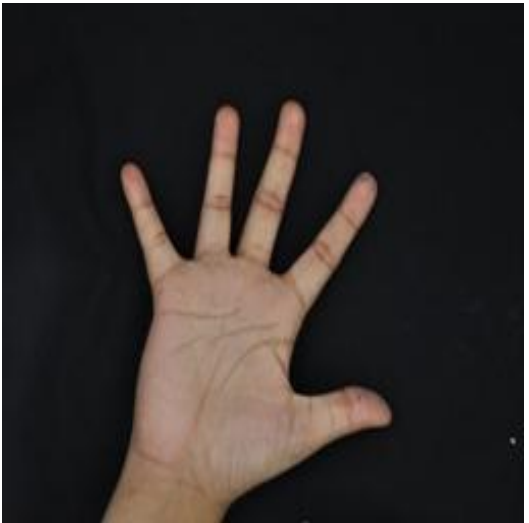

Figure 3 Input Hand image

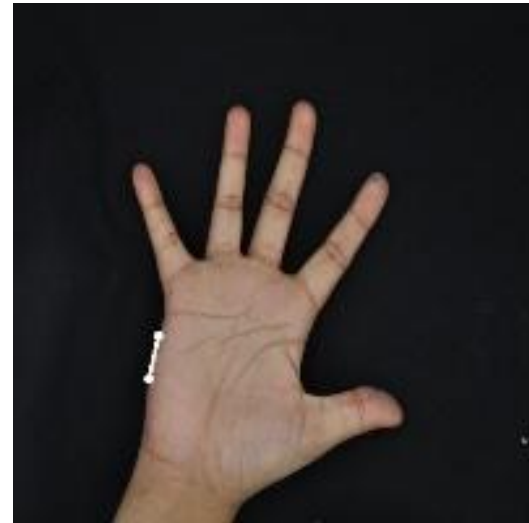

Figure 4 Identification of Left edge of hand

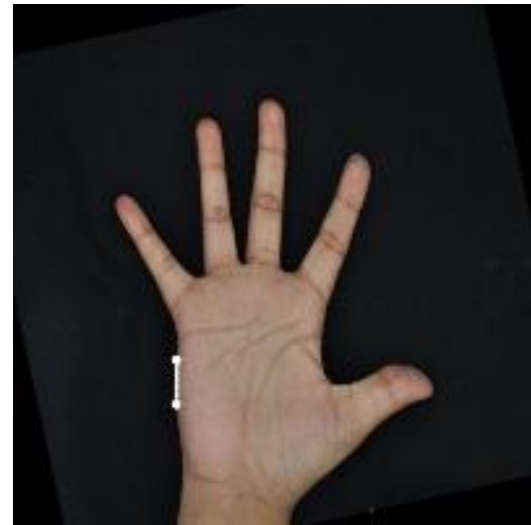

Figure 5 Rotated Image
Step 3 For the points considered in step 2 condition 1 and condition 2 are applied to check whether it satisfies both the conditions. If yes then all the selected points are stored.

Step 4 Selected points are sorted in ascending order based on their x coordinate values.

Step 5 Sorted points are grouped into three groups by comparing $\mathrm{x}$ coordinate values of consecutive points (illustrated in figure 7).

Step 6 The average $\mathrm{x}$ and $\mathrm{y}$ coordinate values are computed in each group. These average values form the valley points.

\subsubsection{Finger tip detection algorithm}

Condition 3. Considering $\mathrm{p}$ as centre of circle and using midpoint circle generation algorithm points along the circumference of circle with radius 10 are found. If number of consecutive points lying in the non hand region is more than seventy percent of the total points on the circumference of the circle, then point $\mathrm{p}$ is considered for further process otherwise the algorithm continues to check the next point along contour.

Algorithm 2: Input: Binary image, valley points. Output: Finger tip points

Step 1 Two valley points which are on either side of finger under consideration are selected.

Step 2 The contour along the selected valley points is traversed.

Step 3 While traversing along the contour path the point under consideration is verified to check whether it satisfies condition 3 . If yes then corresponding point is selected.

Step 4 Selected points are sorted in ascending order based on the count of number of consecutive points lying in the non hand region denoted by $\mathrm{n}$. The point with maximum count is considered as tip point of the corresponding finger. Value of $\mathrm{n}$ considered is as 30 .

Step 5 Steps 1 to 4 are applied for little, ring, middle and index finger.

Finger tip points and valley points identified are shown in figure 8 as filled circles.

\subsection{ROI extraction}

The valley points found using the valley detection algorithm serve as the base points to locate the palm print region and the tip point along with valley points serve as base points to locate IKP ROI.

\subsubsection{Palm print ROI}

For cropping palm print ROI following steps are used.

i) Two key points are selected from the set of nine key points identified. First point is the valley point between little finger and ring finger, second point is the valley point between middle finger and index finger. These points are considered as anchor points.

ii) ROI of palm print is the rectangle region selected using the anchor points. The width of ROI is considered as the distance between anchor points and left top corner of rectangular region is selected as 30 pixels just below the first anchor point. (illustrated in figure 9).

The ROI part containing the palm print is cropped out of the main image and then resized to a size of $128 \mathrm{X} 128$ pixels. The resized palm print ROI image undergoes no further preprocessing. Extracted ROI of palm print is shown in figure 10.

\subsubsection{IKP ROI}

The second knuckle region of finger is found at For cropping IKP ROI of finger following steps are used.

i) Two valley points on either side of finger under consideration are selected as anchor points.

ii) Tip of the finger is considered as the third anchor point.

iii) Finger length $F$ is calculated as the distance between finger tip and midpoint of anchor points identified in step 1.

iv) Second knuckle region located between $1 / 2$ to $3 / 4$ the length of the finger identified.

v) A rectangular area lying inside the contour of the finger in the region bounded at second knuckle region of finger identified in step iv is considered as IKP ROI.

The ROI part containing the IKP is cropped out of the finger region (illustrated in figure 11). Cropped IKP ROI of middle finger is shown in figure 12. Its size is normalized by resizing the ROI to a size of 40 X 150 pixels using bi cubic interpolation.

\section{EXPERIMENTAL RESULTS}

In order to evaluate the proposed approach, verification experiments were conducted on the collected database and in this work IKP RCOI of middle finger is considered for experimental purpose. 


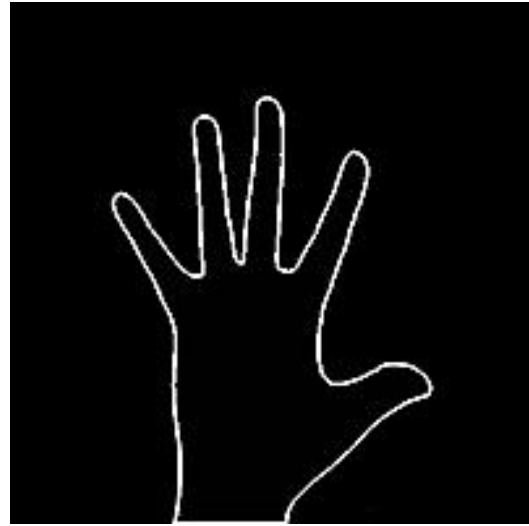

Figure 6 Contour of hand image

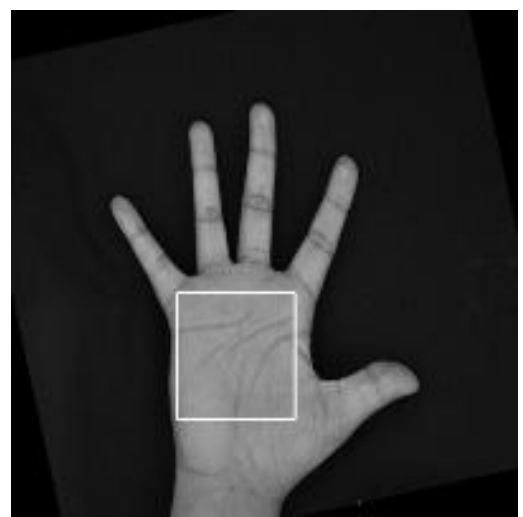

Figure 9 Identified Palm print ROI Location

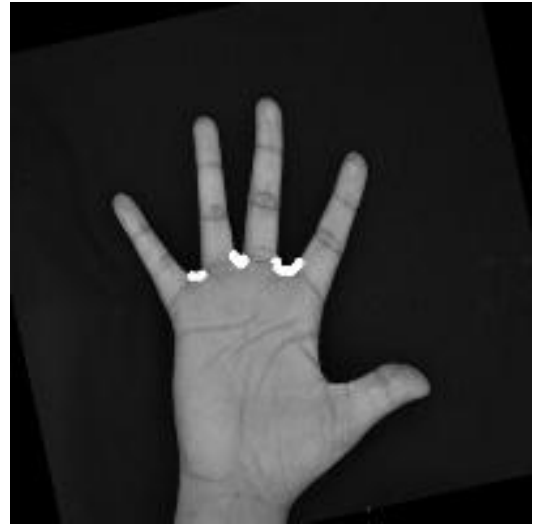

Figure 7 Valley point identification process

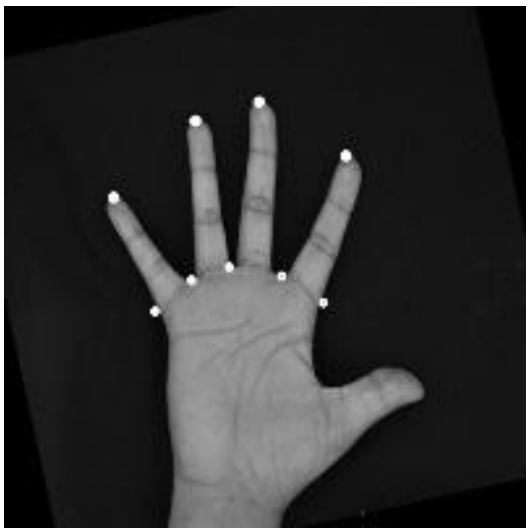

Figure 8 Identified Valley and finger tips points

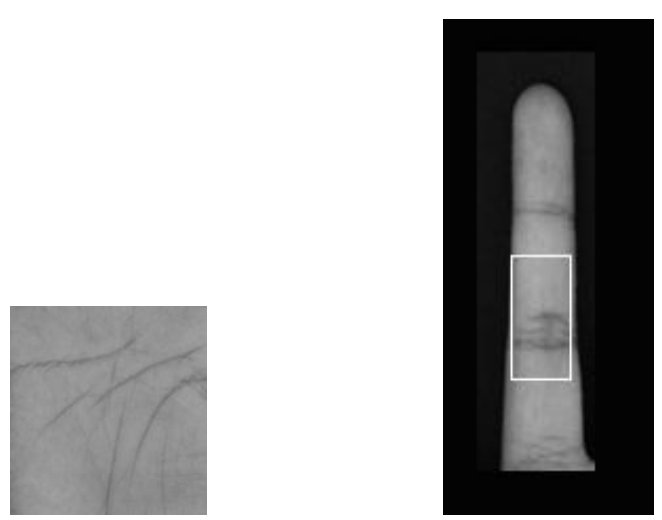

Figure 10 Palm print ROI
Figure 11 Identified IKP ROI location

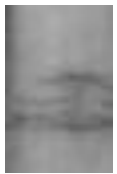

Figure 12 IKP ROI
Complete image database is divided into two mutually exclusive gallery (training) and probe (test) set. The gallery set consists of images with known identities. However, probe set consists of images whose identity is to be known. Images of first session were considered as gallery set and second session images as probe set. The matching of the image pair (gallery and probe image) is done by computing the distance between the two feature vectors. The Euclidean distance metric is used as it achieves good results at low computational cost. Euclidean distance $d$ between the two feature vectors is defined as

$$
d(P, G)=\sqrt{\sum_{i=1}^{n}\left(P_{i}-G_{i}\right)^{2}}
$$

Where $\mathrm{P}$ and $\mathrm{G}$ are feature vectors to be compared and $\mathrm{n}$ refers to the dimension of feature vector. Local binary pattern (LBP) [12] has been widely used in biometric recognition system to extract texture information from biometric images. The LBP operator assigns a binary label to every pixel in the image by thresholding it against the eight neighborhood pixels. If the pixel's value is greater than the neighbor pixel value then binary value 1 is assigned, otherwise 0 is assigned. A binary label is called uniform if it consists of at most two bit-wise transitions from 0 to 1 or vice-versa. For example 11101111 and 10111111 are uniform binary labels where as 10101111 and 01011011 are non-uniform. There are 58 labels of uniform patterns and the rest 198 labels are of non-uniform. For each pixel in the image the LBP operator is extracted. Image borders are ignored due to the fact that a $3 \times 3$ neighborhood is required. A label is given to each of the uniform patterns, and the other non-uniform patterns are assigned to a single label. After the labels have been determined, a histogram $\mathrm{H}_{l}$ of the labels is constructed as:

$$
H_{l}=\sum\{L(i, j)=l\}, l=0, \ldots, q-1
$$

Where $\mathrm{q}$ is the number of different labels produced by the LBP operator, while $\mathrm{i}$ and $\mathrm{j}$ refers to the pixel location. LBP histograms calculated from palm print ROI and IKP ROI are stored as feature vectors of 59 dimensions each. The larger the distance between the two feature vectors the more dissimilar are the two images. At the verification stage a threshold $\mathrm{T}$ is used to regulate the system decision at matching stage. If the Euclidean distance between pair of feature vector is less than or equal to $\mathrm{T}$, then they are considered to belong to the same individual else they are considered to belong to different individuals. In the verification experiment equal error rate(ERR) [13-15] is adopted to evaluate the performance of biometric systems. ERR is the crossing point between false acceptance rate (FAR) and false rejection rate(FRR). FAR is the percentage of authorized individuals that the biometric system fails to accept and FRR is the percentage of unauthorized individual that the biometric system fails to reject. The result expressed as FAR and FRR depending on the threshold is plotted as shown in figure 13 and figure 14 for palm print and IKP respectively. FAR and FRR are closely related since they both depend on the acceptance threshold which is set according to the requirement of the desired 
security level. So threshold is set depending on whether a low FAR or a low FRR is needed.

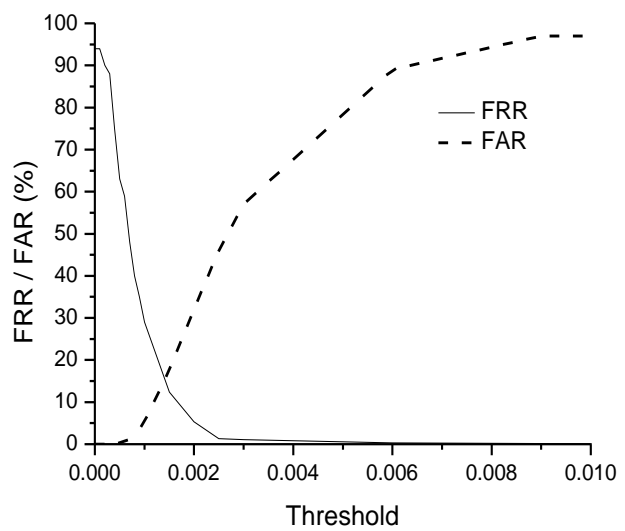

Figure 13 The curve of FAR and FRR against the threshold for palm print

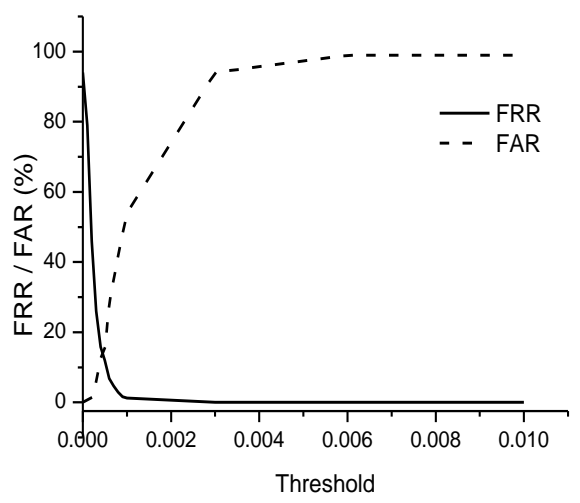

Figure 14 The curve of FAR and FRR against the threshold for IKP

\section{CONCLUSIONS}

This paper proposes a new approach of selecting palm print and IKP ROI suitable for feature extraction that can be utilized in developing biometric recognition systems. Proposed approach is of significance since hand geometry and palm print features can be extracted from the palm region of the hand. The effectiveness of the proposed ROI extraction approach has been tested on the database collected at our institute with a texture based verification system. The obtained results clearly indicate that both traits can be used in developing biometric recognition systems. By reducing the ERR value security of the biometric recognition system can be enhanced. Since both the traits are rich in texture pattern and less effected by aging as future work authors would like to explore to what extent the fusion of palm print and IKP traits reduces the ERR to enhance the security level of biometric recognition systems.

\section{REFERENCES}

[1] Anil K .Jain, "An Introduction to Biometric recognition", IEEE transactions on circuits and systems for video technology, vol. 14, no. 1, pp 1-20,January 2004.
[2] A.Ross and Anil K Jain,"Multimodal biometrics an overview", Proceedings of 12th European Signal Processing Conference,Poland, pp1121-1124, September 2007.

[3] Kresimir Delac, Mislav Grgic, “ A survey of biometric recognition methods", $46^{\text {th }}$ International Symposium Electronics in Marine, pp 184-193,June 2004.

[4] C. Poon, D.C.M. Wong, H.C. Shen, "A New Method in Locating and Segmenting Palmprint into Region of Interest", Proceedings of the 17th International Conference on Pattern Recognition, ICPR 2004, Vol.4 pp 533 - 536 , 23-26 Aug. 2004

[5] Chin-Chuan Han, Hsu-Liang Cheng, Chih-Lung Linb, Kuo-Chin Fan "Personal authentication using palm-print features", Pattern Recognition vol 36 (2003), pp 371 381.

[6] Tee Connie, Andrew Teoh, Beng Jin, Michael GohKahong, David Ngo Chek Ling, "An automated palm print recognition system," Image and Vision Computing No 23 (2005), pp 501-515 .

[7] Zohaib Khan, Faisal Shafait, Yiqun Hu, Ajmal Mian, "Multispectral palm print encoding and recognition", eprint, arXiv:1402.2941v1, 6 Feb, 2014.

[8] Zhu Le qing, Zhang San yuan, "Multimodal biometric identification system based on finger geomerty, knuckle print and palm print", Pattern Recognition Letters, No 31, pp 1641-1649,2010.

[9] Goh Kah Ong Michael, Tee Connie, Andrew Beng Jin Teoh, "Touch less palm print biometrics : A Novel design and implementation" Image and Vision Computing No 36, pp 1550-1560,2008.

[10] Qiang li, Zhengding Qiu, Dongmei Sun and Jie wu "Personal Identification Using Knuckle print", Proceedings of the 5th Chinese conference on Advances in Biometric Person Authentication, Lecture notes in Computer science, volume 3338, Pages 680-689,2005.

[11] Xuemiao Xu, Qiang Jin, Le Zhou, Jing Qin, Tien-Tsin wong, Guoqiang Han, "Illumunation- Inavrient and Deformation-Tolerant Inner knuckle Print Recognition Using Portable Devices",, No 15, pp 4326-4352, Sensors 2015.

[12] T.Ojala, M. Pietikainen and T. Maenpaa, "Multiresolution gray-scale and rotation invariant texture classification with local binary patterns", IEEE Transaction on Pattern Analysis and Machine Intelligence, vol. 27(7), pp. 971-987, 2002.

[13] Kamalesh Tiwari, Devandra K Arya, G.S. Badrinath, Phalguni Gupta, "Designinig palm print based recognition system using local structure tensor and force field transformation for human identification", Neurocomputing, No 116, pp222-230,2013.

[14] Lin Zhang, Lei Zhang, David Zhang, Zhenhua Guo, "Phase congruency induced local features for finger knuckle print recognition", Pattern Recognition No 45, pp 2522-2531, 2012.

[15] M.P.Dale, M.A, Joshi, H.J. Galiyawala, "A single sensor HAnd geometry and palm texture fusion for person identification", International Journal of Computer Applications, Vol 42, No 7,pp11-15, March 2012. 